\title{
Strain-induced time-reversal odd superconductivity in graphene
}

\author{
Bitan Roy ${ }^{1,2}$ and Vladimir Juričić 3,4 \\ ${ }^{1}$ National High Magnetic Field Laboratory and Department of Physics, Florida State University, Florida 32306, USA \\ ${ }^{2}$ Condensed Matter Theory Center, Department of Physics, University of Maryland, College Park, Maryland 20742, USA \\ ${ }^{3}$ Institute for Theoretical Physics, Utrecht University, Leuvenlaan 4, 3584 CE Utrecht, The Netherlands \\ ${ }^{4}$ Instituut-Lorentz for Theoretical Physics, Universiteit Leiden, P.O. Box 9506, 2300 RA Leiden, The Netherlands
}

(Received 4 November 2013; revised manuscript received 14 July 2014; published 30 July 2014)

\begin{abstract}
Time-reversal symmetry breaking superconductors are exotic phases of matter with fascinating properties, which are, however, encountered rather sparsely. Here we identify the possibility of realizing such a superconducting ground state that exhibits an $f+i s$ pairing symmetry in strained graphene. Although the underlying attractive interactions need to be sufficiently strong and comparable in pristine graphene to support such pairing state, we argue that strain can be conducive for its formation even for weak interactions. We show that quantum-critical behavior near the transition is controlled by a multicritical point, characterized by various critical exponents computed here in the framework of an $\epsilon$ expansion near four space-time dimensions. Furthermore, a vortex in this mixed superconducting state hosts a pair of Majorana fermions supporting a quartet of insulating and superconducting orders, among which is the topologically nontrivial quantum spin Hall insulator. These findings suggest that strained graphene could provide a platform for the realization of exotic superconducting states of Dirac fermions.
\end{abstract}

DOI: 10.1103/PhysRevB.90.041413

PACS number(s): 72.80.Vp, 05.30.Rt, 71.10.Fd, 81.05.ue

The time-reversal-symmetry (TRS) breaking superconducting states are exotic phases of quantum matter, and often arise from the competition of two pairings that break distinct lattice or continuous symmetries. The proposed realizations of such states are rather sparse and some of the well-studied examples in two spatial dimensions are the $d+i d$ pairing, discussed in the context of high- $T_{c}$ superconductivity [1], graphene [2,3], and $p+i p$ pairing in fractional quantum Hall systems [4]. A realization of the chiral, time-reversal odd $f$-wave pairing has been proposed in a hole-doped semiconductor, interfaced with a conventional superconductor and a magnetic insulator [5]. Recently it has been argued that a parity and time-reversal odd axionic $p+i s$ pairing state can be realized in weakly correlated, strong spin-orbit coupled three-dimensional doped narrow gap semiconductors [6], such as $\mathrm{Sn}_{1-x} \mathrm{In}_{x} \mathrm{Te}$ [7] and $\mathrm{Cu}_{x} \mathrm{Bi}_{2} \mathrm{Se}_{3}$.

The low-energy electron excitations in graphene, effectively described by the pseudorelativistic Dirac equation [8], can host a plethora of broken-symmetry phases if the interactions are sufficiently strong [9]. Interestingly enough, it is also possible, at least in principle, to realize various relativistic superconducting orders if the net electron-electron interaction acquires an attractive component, which may be induced by the proximity effect or electron-phonon interaction [10]. For example, a strong on-site (next-nearest-neighbor) attractive interaction $U\left(V_{2}\right)$ supports a spin-singlet (-triplet) $s(f)$-wave superconductor [11,12] [see Fig. 1 (left)]. Unconventional spatially inhomogeneous superconducting states have also been proposed for the honeycomb lattice when the nearestneighbor pairing interaction is strong enough [10].

Strain when combined with proximity effect may provide an ideal setting for realizing superconductivity in graphene. The effect of the strain or buckling can be captured by a time-reversal-symmetric axial magnetic field [13,14], which irrespective of its spatial profile, always brings a large number of states at zero energy $[13,15]$. Henceforth, even sufficiently weak attractive interactions can give rise to pairings $[16,17]$.
These special, and topologically protected flat band at zeroenergy in strained graphene [18] live on one sublattice in the bulk, while those residing on the other sublattice can only be found near the boundary of a finite graphene system $[15,19]$. Therefore, application of strain to half-filled graphene in proximity of a superconductor naturally selects only the intrasublattice $s$ - and $f$-wave pairings, since the pairing occurs exclusively among the lowest energy states which, in turn, reside on only one sublattice $[11,12]$. The remaining two fully gapped paired states, namely, the Kekule superconductors [10], couple two sublattices and are therefore excluded. One can thus induce a competition between $s$ - and $f$-wave pairings by placing a strained graphene flake in proximity to a regular $s$-wave, as $\mathrm{Nb}$, and an unconventional odd-parity triplet superconductor, e.g., $\mathrm{UPt}_{3}$, upon suppressing residual weak repulsive interactions at Dirac points $[9,10,20]$. Several questions then arise in this physical context: (i) What is the nature of the pairing symmetry of the ultimate superconducting state resulting from the competition of the $s$ - and $f$-wave pairings? (ii) What is the effective field theory that captures the phase transition when these two pairings compete? (iii) How does the axial field influence such transition, besides being its catalyst?

We here address the competition between $s$ - and $f$-wave pairings and the emergent superconducting multicriticality using a perturbative $\epsilon(=4-d)$ expansion close to the upper critical $d=(3+1)$ space- (imaginary-) time dimensions [21,22] of the effective Gross-Neveu-Yukawa theory. This theory contains both Lagrangians describing the quantum criticality in the vicinity of these two transitions separately, as well as incorporates the coupling of these two order parameters (OPs). When the pairing interactions in the two channels are comparable, a TRS breaking $f+i s$ or $s+i f$ state emerges at low temperatures [see Fig. 1 (right)]. In contrast to a pure bosonic system [23-25], transition to either of these two states is governed by a $Z_{2} \otimes O(3)$ symmetric mixed Gross-Neveu-Yukawa multicritical point due to the nontrivial 

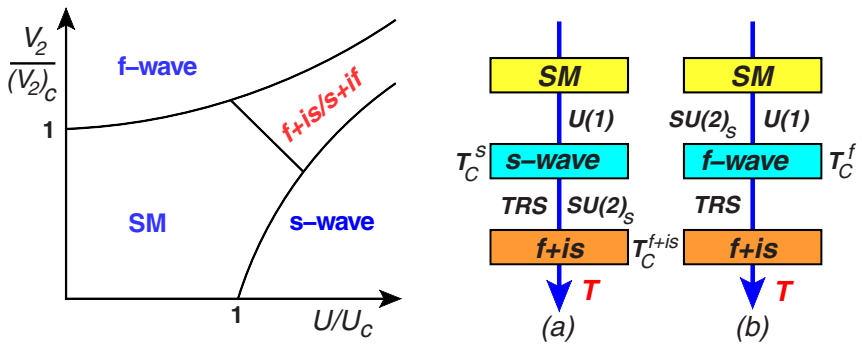

FIG. 1. (Color online) Left: A schematic $T=0$ phase diagram with on-site $(U)$ and next-nearest-neighbor $\left(V_{2}\right)$ attractions. $U_{c}$ and $\left(V_{2}\right)_{c}$ are the critical strength of pairing interactions driving superconducting instabilities of the Dirac semimetal (SM). Right: Two possible scenarios for two-stage transitions at finite temperatures: (a) when the $s$-wave transition temperature $T_{C}^{s}>T_{C}^{f}$; (b) when the $f$-wave transition temperature $T_{C}^{f}>T_{C}^{s}$ (temperature decreases in the direction of the arrow). In the cascade transitions global charge $[U(1)]$, spin-rotation symmetries $\left[S U(2)_{s}\right]$, and TRS are broken, as shown in the figure.

Yukawa couplings, resembling in this regard the situation near an insulating quantum critical point of Dirac fermions [26]. The scaling of the pairing amplitudes with the axial field is essentially governed by a set of critical exponents computed in its absence. The recent surge of experimental works to realize and tune the strain-induced axial magnetic field in real and artificial graphene [27-29], observation of proximity induced superconductivity in graphene resting on metallic rhenium, with $T_{C} \sim 2.1 \mathrm{~K}$ [30], and search for the exotic broken-symmetry phases in this setup $[15,16,31]$ make our study important, timely, and experimentally pertinent.

The dynamics of the free Dirac fermions, living around $\pm \mathbf{K}$ points, where $\mathbf{K}=(1,1 / \sqrt{3})(2 \pi / a \sqrt{3})$, with $a \approx 3 \AA$ as the lattice constant, is captured by a relativistically invariant Lagrangian $L_{f}=\bar{\Psi}(x) \sigma_{0} \otimes \gamma_{\mu} \partial_{\mu} \Psi(x)$. The eight-component Dirac-Nambu spinor, invariant under the spin rotations, generated by $\vec{S}=\vec{\sigma} \otimes I_{4}$, is defined as $\Psi^{\dagger}(k)=\left[\Psi_{+}^{\dagger}(k), \Psi_{-}^{\dagger}(k)\right]$, where $\Psi_{\sigma}^{\dagger}(k)=\left[u_{\sigma}^{\dagger}(k), v_{\sigma}^{\dagger}(k), \sigma u_{-\sigma}(-k), \sigma v_{-\sigma}(-k)\right]$. Here, $k \equiv(\omega, \mathbf{k})$ is the three-momentum, $\mathbf{k}=\mathbf{K}+\mathbf{q},|\mathbf{q}| \ll|\mathbf{K}|$, and summation over repeated indices is assumed. $\sigma= \pm$ are the spin projections along the $z$ axis; $u_{\sigma}, v_{\sigma}$ are the spinor components on the two sublattices; and as usual $\bar{\Psi} \equiv \Psi^{\dagger} \sigma_{0} \otimes \gamma_{0}[32]$. The real $(\varphi)$ and the imaginary $(\chi)$ parts of the $s$-wave OP are defined as

$$
\Phi(x)=\left\langle\bar{\Psi}(x) \sigma_{0} \otimes\left(I_{4} c \theta+i \gamma_{5} s \theta\right) \Psi(x)\right\rangle \equiv \varphi(x)+i \chi(x),
$$

after rotating the spinor as $\Psi \rightarrow U \Psi$ with $U=\exp \left[i \frac{\pi}{4} \sigma_{0} \otimes\right.$ $\left.\gamma_{3}\right], c \equiv \cos$, and $s \equiv \sin$. Such rotation leaves $L_{f}$ invariant and allows the extension of the theory from the physical $(2+1)$ to $(3+1)$ dimensions. The coupling of the $s$-wave OP with the gapless Dirac fermions assumes the form of the Yukawa interaction

$$
L_{b-f}^{s}=g_{1 s} \varphi\left(\bar{\Psi} \sigma_{0} \otimes I_{4} \Psi\right)+g_{2 s} \chi\left(\bar{\Psi} \sigma_{0} \otimes i \gamma_{5} \Psi\right),
$$

with $g_{1 s / 2 s} \sim U$. The effective theory describing the transition into the $s$-wave superconductor is given by $L^{s}=L_{f}+$

$$
\begin{aligned}
& L_{b-f}^{s}+L_{b}^{s}, \text { with } \\
& L_{b}^{s}=\sum_{\alpha=\varphi, \chi}\left[\frac{1}{2}\left(\partial_{\mu} \alpha\right)^{2}+m_{\alpha}^{2} \alpha^{2}+\frac{\lambda_{\alpha}}{4 !} \alpha^{4}\right]+\frac{\lambda_{\varphi \chi}}{12} \varphi^{2} \chi^{2},
\end{aligned}
$$

as the Ginzburg-Landau Lagrangian describing the dynamics of the singlet OP. The superconducting mass $m_{\alpha}^{2} \sim\left(U-U_{c}\right)$, where $U_{c}$ is the zero-axial-field critical on-site attraction for $s$-wave ordering. We here allow a generic situation where the Yukawa and the bosonic couplings for the real and the imaginary parts of the OP are different. As we show near the multicritical point for the transition into the $f+i s(s+i f)$ state, only one Yukawa and bosonic quartic couplings are nontrivial.

Analogously, the effective theory $L^{t}=L_{f}+L_{b-f}^{t}+L_{b}^{t}$, defined below, describes the universal behavior near the transition into the triplet $f$-wave pairing. The real $(\vec{\varphi})$ and imaginary $(\vec{\chi})$ part of the $f$-wave OP $\vec{\Phi}(x) \equiv \vec{\varphi}(x)+i \vec{\chi}(x)$, is obtained from Eq. (1) by replacing $\sigma_{0}$ by $\vec{\sigma}$. This OP is even (odd) under the sublattice (valley) exchange [10,32], and its coupling with the Dirac fermions again assumes the Yukawa form

$$
L_{b-f}^{t}=g_{1 t} \vec{\varphi} \cdot\left(\bar{\Psi} \vec{\sigma} \otimes I_{4} \Psi\right)+g_{2 t} \vec{\chi} \cdot\left(\bar{\Psi} \vec{\sigma} \otimes i \gamma_{5} \Psi\right),
$$

where $g_{1 t / 2 t} \sim V_{2}$. The dynamics of the triplet OP is described by the Lagrangian

$$
L_{b}^{t}=\sum_{\vec{\alpha}=\vec{\varphi}, \vec{\chi}}\left[\frac{1}{2}\left(\partial_{\mu} \vec{\alpha}\right)^{2}+m_{\vec{\alpha}}^{2} \vec{\alpha}^{2}+\frac{\lambda_{\vec{\alpha}}}{4 !}(\vec{\alpha} \cdot \vec{\alpha})^{2}\right]+\frac{\lambda_{\vec{\varphi}} \vec{\chi}}{12} \vec{\varphi}^{2} \vec{\chi}^{2},
$$

a generalization of the singlet version in Eq. (3), with $m_{\vec{\alpha}}^{2} \sim V_{2}-\left(V_{2}\right)_{c}$, where $\left(V_{2}\right)_{c}$ is the zero-axial-field critical interaction for $f$-wave pairing. Also here we allow for all the bare couplings to be different.

The coupling of the singlet and the triplet OPs close to the multicritical point is given by

$L_{b}^{s t}=\frac{\lambda_{\varphi \vec{\varphi}}}{12} \varphi^{2} \vec{\varphi}^{2}+\frac{\lambda_{\chi \vec{\chi}}}{12} \chi^{2} \vec{\chi}^{2}+\frac{\lambda_{\varphi \vec{\chi}}}{12} \varphi^{2} \vec{\chi}^{2}+\frac{\lambda_{\chi \vec{\varphi}}}{12} \chi^{2} \vec{\varphi}^{2}$,

and their competition is described by the Lagrangian $L=$ $L_{f}+L_{b}^{s}+L_{b-f}^{s}+L_{b}^{t}+L_{b-f}^{t}+L_{b}^{s t}$. Throughout this Rapid Communication, we omit the couplings of the massless Dirac fermions and the superconducting OPs with the fluctuating gauge fields, since both the Fermi and the bosonic velocities are much smaller than the velocity of light. The ultimate critical behavior is, however, governed by a charged critical point, where all the velocities are equal, although such deep infrared critical behavior may not be accessible experimentally due to their logarithmically slow increase [33].

Since all the Yukawa and the quartic bosonic couplings in this theory are exactly marginal in $d=(3+1)$, we use the $\epsilon$ expansion about four dimensions, with $\epsilon=4-d$, as the tool for studying the quantum-critical behavior. The standard minimal-subtraction scheme [21,34] yields the (infrared) $\beta$ functions for the Yukawa couplings $g_{1 s}, g_{1 t}$,

$$
\begin{aligned}
& \beta_{g_{1 s}^{2}}=\epsilon g_{1 s}^{2}-(2 N+3) g_{1 s}^{4}+g_{1 s}^{2}\left(g_{2 s}^{2}-9 g_{1 t}^{2}+3 g_{2 t}^{2}\right), \\
& \beta_{g_{1 t}^{2}}=\epsilon g_{1 t}^{2}-(2 N+1) g_{1 t}^{4}-g_{1 t}^{2}\left(5 g_{2 t}^{2}+3 g_{1 s}^{2}-g_{2 s}^{2}\right),
\end{aligned}
$$


after taking $N_{d} g^{2} \rightarrow g^{2}$, where $N_{d} \equiv S_{d} /(2 \pi)^{d}$ and $S_{d} \equiv$ $2 \pi^{d / 2} / \Gamma(d / 2)$, with $N(=2$ for graphene) as the number of four-component Dirac fermions. The $\beta$ functions for the other two Yukawa couplings, $g_{2 s}$ and $g_{2 t}$, are obtained by replacing $1 \leftrightarrow 2$ in the above two $\beta$ functions. Interestingly, the $\beta$ functions for the Yukawa couplings are decoupled from the bosonic quartic couplings $\left(\lambda_{\alpha}\right)$, and out of 16 fixed points in the four-dimensional subspace spanned by the Yukawa couplings, only two are fully stable. One that describes the transition into the $f+i s$ state is located at

$$
\left(g_{2 s}^{*}\right)^{2}=\left(g_{1 t}^{*}\right)^{2}=\frac{\epsilon}{2 N}, \quad g_{1 s}^{*}=g_{2 t}^{*}=0,
$$

while the other one that corresponds to the transition into the $s+i f$ state is obtained by replacing $1 \leftrightarrow 2$ above. Therefore, our renormalization group analysis suggests that when singlet and triplet pairings compete in the system of massless Dirac fermions, the transition is always multicritical in nature and towards the formation of a mixed $f+i s$ or $s+i f$ state, with TRS dynamically broken due to the Yukawa interactions (see Fig. 1).

This outcome can further be substantiated from the minimization of free energy. The effective single-particle Hamiltonian with both singlet and triplet OPs reads

$$
H=\sigma_{0} \otimes i \gamma_{0} \vec{\gamma} \cdot \vec{p}+\sum_{x=s, f} \Delta_{x} \sigma_{x} \otimes \gamma_{0}\left(\cos \theta_{x}+i \gamma_{5} \sin \theta_{x}\right)
$$

where $\sigma_{s / f}=\sigma_{0 / 3}, \vec{p}$ is the momentum operator, and we fix the spin quantization of the $f$-wave OP along the $z$ axis, for simplicity. Its spectrum contains two branches of positive energy

$$
E_{ \pm}=\sqrt{p^{2}+\Delta_{s}^{2}+\Delta_{f}^{2} \pm 2 \Delta_{s} \Delta_{f} \cos \left(\theta_{s}-\theta_{f}\right)},
$$

and the corresponding negative ones at $-E_{ \pm}$. Therefore, the energy of the filled Dirac-Fermi sea at half filling $-\left(E_{+}+\right.$ $\left.E_{-}\right)$, is maximally lowered when $\theta_{s}-\theta_{f}= \pm \pi / 2$. All the terms in Eq. (9) then enter as the sum of the squares in the expression of energy, and the Dirac points are maximally gapped. This constraint corresponds to two exactly degenerate TRS breaking $f+i s$ and $s+i f$ states, as we have found from the above renomalization group calculation, suggesting its robustness against fluctuations. In turn, this gives confidence that our result could be valid beyond the leading order in the $\epsilon$ expansion.

Near the Yukawa fixed point in Eq. (8), where only the imaginary (real) part of the $s(f)$-wave order-parameter is nonvanishing, three bosonic quartic couplings $\left(\lambda_{\chi}, \lambda_{\vec{\varphi}}, \lambda_{\chi \vec{\varphi}}\right)$ are nontrivial, yielding a $Z_{2} \otimes O(3)$ symmetric critical theory. Their $\beta$ functions yield only one fully stable fixed point, which for $N=2$ (graphene) in the critical plane $\left(m_{\chi}=m_{\vec{\varphi}}=0\right)$ is located at $\left(\lambda_{\chi}^{*}, \lambda_{\vec{\varphi}}^{*}, \lambda_{\chi \vec{\varphi}}^{*}\right)=(0.972,0.992,1.103) \epsilon$ [34]. Hence, the transition to the $f+i s(s+i f)$ state is described by a fermionic mixed critical point, where besides the Yukawa couplings of the singlet and the triplet OPs, their quartic interactions as well as their mutual coupling are finite. In the absence of Yukawa couplings, two fully stable fixed points in a $Z_{2} \otimes O(3)$ purely bosonic theory are decoupled $\left(\lambda_{\chi \vec{\varphi}}=0\right)$.
They are located at $\left(\lambda_{\chi}, \lambda_{\vec{\varphi}}\right)=(2 \epsilon / 3,0)[(0,6 \epsilon / 11)]$, and describe the transition into the pure singlet [triplet] phase. Since the anomalous dimension of the bosonic fields $\eta_{\vec{\varphi}}(=$ $\left.0.020 \epsilon^{2}\right)>\eta_{\chi}\left(=0.018 \epsilon^{2}\right)$ [25], the ultimate criticality in the purely bosonic theory is possibly governed by the triplet critical point [35]. In contrast, the massless fermions, through Yukawa couplings to the critical bosonic fluctuations, stabilize the mixed multicritical point in graphene governing the transition into the $f+i s(s+i f)$ state. The bicritical fixed points in our theory lie in the unphysical regime of couplings $(\lambda$ 's $<0)$. Therefore, the transition into the $f+i s(s+i f)$ state is always continuous in nature.

The critical theory possesses two relevant operators, the masses $m_{\chi}$ and $m_{\vec{\varphi}}$, that tune the phase transition into the mixed state. Their flow defines the correlation-length exponents $\left(v_{\chi}, v_{\vec{\varphi}}\right)=(1 / 2+0.509 \epsilon, 1 / 2+0.521 \epsilon)$ in the vicinity of the above multicritical point. The anomalous dimensions for spinsinglet/triplet OP and Dirac fermions close to the transition is $\eta_{\chi / \vec{\varphi}}=\epsilon$ and $\eta_{\Psi}=\epsilon / N$, respectively [34]. Since the mass in the $f+i s$ state is Lorentz symmetric, we expect weak Lorentz-symmetry-breaking perturbations to be irrelevant and the dynamical critical exponent $z=1$, close to this critical point [22]. The residue of the quasiparticle pole vanishes as $m_{\alpha}^{z v_{\alpha} \eta_{\Psi}}$ with $\alpha=\chi, \vec{\varphi}$, depending on the relevant direction from which the $f+i$ s state is approached, and Dirac fermions cease to exist as sharp quasiparticle excitations at the transition. The critical exponents near the superconducting multicritical point are different than the ones in the vicinity of the pure $s$ wave transition, where the correlation-length exponent is $v=$ $1 / 2+0.3 \epsilon$, and fermionic (bosonic) anomalous dimension is $\eta_{\Psi}=4 \epsilon / 6\left(\eta_{b}=\epsilon / 6\right)$ [33].

The quantum-critical behavior near the transition into a pure $f$-wave superconducting state in graphene is described by a critical point located at $\left(g_{t}^{2}, \lambda_{t}\right)=(0.1,0.498) \epsilon$, where $g_{t} \equiv$ $g_{1 t}=g_{2 t}$ and $\lambda_{t} \equiv \lambda_{\vec{\varphi}}=\lambda_{\vec{\chi}}=\lambda_{\vec{\varphi} \vec{\chi}}$. The critical exponents near this critical point are found to be $v=1 / 2+0.266 \epsilon, \eta_{\Psi}=$ $3 \epsilon / 10$, and $\eta_{b}=2 \epsilon / 5$ and are of a distinct non-mean-field nature [34].

As the temperature is gradually lowered, the system first enters the dominant paired $s(f)$-wave state at temperature $T_{C}^{s}\left(T_{C}^{f}\right)$ [see Fig. 1(right)]. Only at even lower temperature $\left(T_{C}^{f+i s}\right)$ the $f+i s$ state is reached with TRS being broken, which can be confirmed by Kerr rotation measurements [36] This two-stage superconducting transition leads to discontinuities in the specific heat at the two critical temperatures. Since the $f$-wave pairing is also more susceptible to generic disorders, it is also possible that the transition temperature for the $s$-wave pairing is higher than that for the $f$-wave pairing [37], which is devoid of Pauli limiting field [38]. Consequently, the lower critical field $H_{c 1}$ discerns abrupt increment below the second transition temperature $T_{C}^{f+i s}$. Since spin of the $f$-wave order can be flipped by applying a radio frequency signal in a nuclear magnetic resonance experiment, after switching off this signal, as the spin relaxes back to the ground state, a radio frequency signal is emitted.

The above critical exponents govern the scaling of the physical quantities, such as the pairing gap, in the presence of axial fields, which catalyze the pairings without changing the universality class of the pertaining transitions. In weak 
axial fields, the pairing gap $(\Delta)$ exhibits the scaling

$$
\frac{\Delta}{g}=\left(l_{b} / a\right)^{-\beta / v} F\left[\left(l_{b} / a\right)^{1 / v}\left(g-g_{c}\right)\right],
$$

similar as in a finite system of the size $l_{b}$, where the axial magnetic length $l_{b}=\sqrt{\hbar / e b} \sim 100 a$ for $b \sim 100 \mathrm{~T}$, and therefore the continuum description remains justified. Here, $\beta$ is the OP exponent, $g-g_{c}$ measures the deviation from the zero-field critical interaction $\left(g_{c}\right)$, and $F(x)$ is the scaling function $[39,40]$. Standard relations between the critical exponents then yield $2 \beta / v=d+z-2+\eta_{b}=2-$ $\left(\epsilon-\eta_{b}\right)$, in effective $d+z=4-\epsilon$ dimensions, with $\eta_{b}$ as the anomalous dimension of the OP at the zero-field critical point [25]. Different critical exponents near the transition into $s$-wave, $f$-wave, and the $f+i s$ states therefore lead to distinct scalings of the gap and concomitantly their transition temperatures with axial fields, which should serve as a clear signal of the cascade two-stage transition, Fig. 1 (right). Even for weak enough pairing interactions, $\Delta \sim g b \sim T_{C}$ (BCS approximation) in the absence of disorder [39], resulting in sizable pairing gaps when $b \sim 50-300 \mathrm{~T}$ [27-29]. Hence, the proposed unconventional $f+i$ s state can possibly be realized in cleaner graphene samples, with $T_{C}^{f+i s} \sim 1 \mathrm{~K}$, as a recent experiment would suggest [30].

Type-II nature of the superconductors inducing the mixed $f+i s$ state in graphene through proximity allows us to investigate the vortex phase therein. Since the single-particle Hamiltonian in Eq. (9) in the $f+i s$ state represents $t w o$ copies of anisotropic Jackiw-Rossi Hamiltonian [41], a single vortex hosts two Majorana fermions [32], and supports four Dirac masses, $\left\{\sigma_{3} \otimes \gamma_{0}, \sigma_{0} \otimes \gamma_{0}, \sigma_{1} \otimes i \gamma_{0} \gamma_{3}, \sigma_{2} \otimes i \gamma_{0} \gamma_{3}\right\}$, inside the vortex core [32], even when real and the axial magnetic fields are present simultaneously [42]. All four of these masses anticommute with $H$ in Eq. (9), and leave the zero-energy subspace invariant. They respectively represent the charge- density wave (CDW), the $z$ component of the topological spin Hall insulator, and the remaining spin components of the $f$-wave superconductor. It is therefore conceivable to realize $f$-wave pairing inside the vortex core, however with different spin components than in the bulk. On the other hand, the Zeeman coupling, $H_{Z}=g B(\vec{x})\left(\sigma_{3} \otimes I_{4}\right)$, where $g \approx 2$ in graphene and $B(\vec{x})$ represents the magnetic field, supports CDW inside the vortex core of the $f+i s$ state, which can be detected using scanning tunneling microscopy, for instance. In the $f$-wave phase the Majorana states support the easy axis components of the Néel OP, represented by the matrices $\left(\sigma_{1}, \sigma_{2}\right) \otimes i \gamma_{1} \gamma_{2}$, besides the CDW and the quantum spin Hall insulator; the latter is then favored by the Zeeman coupling [32].

To summarize, we here demonstrated the possibility of realizing a TRS breaking paired ground state with $f+i s$ symmetry in strained graphene, and addressed its universal properties. Moreover, our results may be consequential for other condensed-matter systems exhibiting low-energy Dirac quasiparticles, such as structurally similar monolayer silicene [43], surface states of topological insulators, and Weyl semimetals.

The authors would like to acknowledge fruitful discussions with Oskar Vafek, Igor Herbut, and Pallab Goswami. B.R. was supported at the National High Magnetic Field Laboratory by NSF Cooperative Agreement No. DMR-0654118, the State of Florida, and the US Department of Energy. V.J. acknowledges the support of the Netherlands Organization for Scientific Research (NWO). The authors are grateful to Advance Study Group and workshop "Spin Orbit Entanglement: Exotic States of Quantum Matter in Electronic Systems" at the Max-Planck Institute for Complex Systems, Dresden, Germany, where part of this work was finalized.
[1] T. Senthil, J. B. Marston, and M. P. A. Fisher, Phys. Rev. B 60, 4245 (1999).

[2] R. Nandkishore, L. Levitov, and A. Chubukov, Nat. Phys 8, 158 (2012).

[3] M. L. Kiesel, C. Platt, W. Hanke, D. A. Abanin, and R. Thomale, Phys. Rev. B 86, 020507(R) (2012).

[4] N. Read and D. Green, Phys. Rev. B 61, 10267 (2000).

[5] L. Mao, J. Shi, Q. Niu, and C. Zhang, Phys. Rev. Lett. 106, 157003 (2011).

[6] P. Goswami and B. Roy, Phys. Rev. B 90, 041301(R) (2014).

[7] M. Novak, S. Sasaki, M. Kriener, K. Segawa, and Y. Ando, Phys. Rev. B 88, 140502(R) (2013).

[8] G. W. Semenoff, Phys. Rev. Lett. 53, 2449 (1984).

[9] I. F. Herbut, V. Juričić, and B. Roy, Phys. Rev. B 79, 085116 (2009).

[10] B. Roy and I. F. Herbut, Phys. Rev. B 82, 035429 (2010).

[11] E. Zhao and A. Paramekanti, Phys. Rev. Lett. 97, 230404 (2006).

[12] C. Honerkamp, Phys. Rev. Lett. 100, 146404 (2008).

[13] I. F. Herbut, Phys. Rev. B 78, 205433 (2008).
[14] F. Guinea, B. Horovitz, and P. Le Doussal, Phys. Rev. B 77, 205421 (2008).

[15] B. Roy and I. F. Herbut, Phys. Rev. B 88, 045425 (2013).

[16] P. Ghaemi, J. Cayssol, D. N. Sheng, and A. Vishwanath, Phys. Rev. Lett. 108, 266801 (2012).

[17] B. Uchoa and Y. Barlas, Phys. Rev. Lett. 111, 046604 (2013).

[18] R. Jackiw and S.-Y. Pi, Phys. Rev. Lett. 98, 266402 (2007).

[19] B. Roy, Ph.D. thesis, Simon Fraser University, 2011, http://theses.lib.sfu.ca/thesis/etd6841.

[20] Repulsive interactions could be overtaken by the pairings in strained graphene near the Dirac point in proximity of a superconductor, since they remain weak upon integrating out fast Fourier modes within the momentum shell $\left(1 / a, 1 / l_{b}\right)$, where $l_{b}$ is the axial magnetic length

[21] J. Zinn-Justin, Quantum Field Theory and Critical Phenomena (Oxford University Press, Oxford, UK, 2002).

[22] I. F. Herbut, V. Juričić, and O. Vafek, Phys. Rev. B 80, 075432 (2009).

[23] P. Calabrese, A. Pelissetto, and E. Vicari, Phys. Rev. B 67, 054505 (2003). 
[24] A. Eichhorn, D. Mesterházy, and M. M. Scherer, Phys. Rev. E 88, 042141 (2013).

[25] I. Herbut, A Modern Approach to Critical Phenomena (Cambridge University Press, Cambridge, UK, 2007).

[26] B. Roy, Phys. Rev. B 84, 113404 (2011).

[27] N. Levy, S. A. Burke, K. L. Meaker, M. Panlasigui, A. Zettl, F. Guinea, A. H. Castro Neto, and M. F. Crommie, Science 329, 544 (2010)

[28] J. Lu, A. H. Castro Neto, and K. P. Loh, Nat. Commun. 3, 823 (2012).

[29] K. K. Gomes, W. Mar, W. Ko, F. Guinea, and H. Manoharan, Nature (London) 483, 306 (2012).

[30] C. Tonnoir, A. Kimouche, J. Coraux, L. Magaud, B. Delsol, B. Gilles, and C. Chapelier, Phys. Rev. Lett. 111, 246805 (2013).

[31] D. A. Abanin and D. A. Pesin, Phys. Rev. Lett. 109, 066802 (2012).

[32] I. F. Herbut, Phys. Rev. Lett. 104, 066404 (2010); Phys. Rev. B 85, 085304 (2012); I. F. Herbut and C.-K. Lu, ibid. 83, 125412 (2011).
[33] B. Roy, V. Juričić, and I. F. Herbut, Phys. Rev. B 87, 041401(R) (2013).

[34] See Supplemental Material at http://link.aps.org/supplemental/ 10.1103/PhysRevB.90.041413 for details of one-loop renormalization group calculation.

[35] E. Vicari and J. Zinn-Justin, New J. Phys. 8, 321 (2006).

[36] A. Kapitulnik, J. Xia, E. Schemm, and A. Palevski, New J. Phys. 11, 055060 (2009).

[37] M. Aizenman and J. Wehr, Phys. Rev. Lett. 62, 2503 (1989); R. Nandkishore, J. Maciejko, D. A. Huse, and S. L. Sondhi, Phys. Rev. B 87, 174511 (2013).

[38] M. Tinkham, Introduction to Superconductivity (Dover, New York, 1996).

[39] I. F. Herbut and B. Roy, Phys. Rev. B 77, 245438 (2008).

[40] F. F. Assaad and I. F. Herbut, Phys. Rev. X 3, 031010 (2013).

[41] R. Jackiw and P. Rossi, Nucl. Phys. B 190, 681 (1981).

[42] B. Roy, Phys. Rev. B 85, 165453 (2012).

[43] L. Chen, B. Feng, and K. Wu, Appl. Phys. Lett. 102, 081602 (2013). 